\title{
Laser threshold of Mie resonances
}

\author{
Karen L. van der Molen and Peter Zijlstra \\ Complex Photonic Systems, MESA+ Research Institute and Department of Science and Technology, \\ University of Twente, P.O. Box 217, 7500 AE Enschede, The Netherlands
}

\section{Ad Lagendijk}

Complex Photonic Systems, MESA+ Research Institute and Department of Science and Technology, University of Twente, P.O. Box 217, 7500 AE Enschede, The Netherlands, and FOM Institute for Atomic and Molecular Physics (Amolf), P.O. Box 41883, 1009DB Amsterdam, The Netherlands

\author{
Allard P. Mosk \\ Complex Photonic Systems, MESA+ Research Institute and Department of Science and Technology, \\ University of Twente, P.O. Box 217, 7500 AE Enschede, The Netherlands
}

\begin{abstract}
Received February 1, 2006; accepted February 20, 2006; posted February 23, 2006 (Doc. ID 67679)
We show that time-independent scattering coefficients calculated from the standard extrapolation of Mie theory to the gain regime have physical meaning up to the laser threshold. The theoretical width of a resonance decreases linearly with increasing gain and becomes zero at the laser threshold. We performed experiments on dielectric microspheres with gain, trapped with optical tweezers. The width of the mode was measured to narrow as a function of the gain up to the lasing threshold, confirming both the validity of the extrapolation of Mie theory to the gain regime below threshold and our interpretation of its point of divergence as the laser threshold. (C) 2006 Optical Society of America
\end{abstract}

OCIS codes: $140.3410,140.4780,290.4020$.

Ideally we would like to investigate laser processes in systems that can be described with rigorous theory and that can be experimentally explored. A small active dielectric sphere, that is, a sphere with gain, constitutes such an ideal system. In this Letter we report a comprehensive, experimental and theoretical, study of the laser properties of a small active dielectric sphere. An impressive body of experiments on microspheres has been successfully described with Mie theory (see, e.g., the selection of Ref. 1 and the recent papers of Refs. 2-6). Mie theory describes the scattering of an incoming continuous plane wave off a dielectric sphere. ${ }^{7}$ Chew has applied this theory to include the scattering of light emitted by a dipole source at any position. ${ }^{8,9}$ Various authors have extrapolated Mie theory to the gain regime (from here on called EMTG) to calculate time-independent scattering coefficients of active spheres, an approach widely used. ${ }^{10-15}$

In this Letter we show that time-independent scattering coefficients calculated from EMTG have physical meaning only if the gain is below a critical value. This critical gain is identified by us as the laser threshold. On the basis of this newly developed insight, we performed experiments on active dielectric microspheres trapped with optical tweezers.

In active spheres (i.e., with gain) laser oscillation can be obtained, and this lasing process occurs predominantly in the morphology-dependent resonances (MDRs) ${ }^{16}$ In EMTG, the gain is introduced as a positive imaginary part in the refractive index of the sphere $^{10-15,17,18}$ (a negative part corresponds to absorption), referred to as the gain index. We have calculated the width of the MDRs for an active sphere, defined for narrow resonances as minus the imaginary part of the pole of the scattering coefficient, ${ }^{19}$ as a function of the gain index. At a critical value of the gain index the width becomes zero, with accompanying divergence of the emission intensity. Exactly at this point, the round-trip amplification can be shown to be equal to one ${ }^{20}$ which means that the mode is at the laser threshold. Above threshold EMTG predicts negative widths; i.e., the poles of the scattering coefficients shift from the lower half to the upper half of the complex plane. This shift has drastic consequences: if the poles are above the real frequency axis, the scattering coefficients (evaluated at real frequencies) no longer have any physical meaning. Specifically, a causal time-independent response function cannot be obtained by the usual Fourier-Laplace transform along the real frequency axis. Various phenomena have been predicted from EMTG, such as broadening of resonance lines and decrease of scattered intensity with increasing gain, ${ }^{10-15}$ all based on the physically meaningless scattering coefficients at real frequency. We stress that for small gain, i.e., up to threshold, the predictions from EMTG are correct. To model the laser at and above threshold one should introduce pump and gain dynamics, thus avoiding any divergence.

A phenomenological formula for the determination of the laser threshold in microspheres has been reported by Campillo et al. ${ }^{5}$ We compare our rigorous criterion with this phenomenological approach for a relevant mode, the transverse electric (TE) mode with mode number 95 . Campillo et al. ${ }^{5}$ used essentially as criterion for lasing that the gain per unit length be larger than the loss per unit length. The amplitude gain per unit length is $2 / l_{g}$, with $l_{g}$ given by $\lambda_{0} / 2 \pi m_{I}^{\prime \prime}$ (with $\lambda_{0}$ the wavelength in vacuum). The complex-valued refractive index inside the microsphere is denoted $m_{I} \equiv m_{I}^{\prime}+i m_{I}^{\prime \prime}$, where $m_{I}^{\prime \prime}$ is the gain 

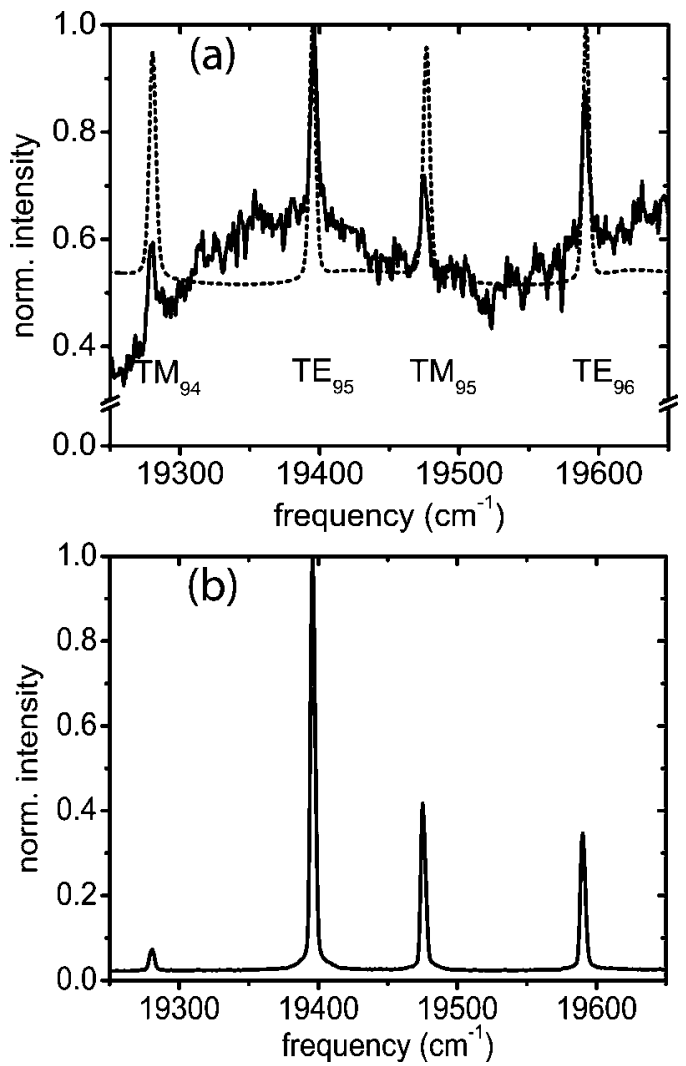

Fig. 1. (a) Experimental emission spectrum of a microsphere excited with a low pumping power of $1 \mathrm{~nJ} / \mathrm{pulse}$ (normalized to the peak intensity of the $\mathrm{TE}_{95}$ mode). The dashed curve shows a theoretical fit of Mie theory. (b) Experimental spectrum of the same sphere measured at $100 \mathrm{~nJ} /$ pulse, above threshold.

index. The gain index threshold calculated by using the model of Campillo et al. lies $7.1 \%$ below our exact value for our parameters. The origin of this discrepancy is well explained by the implicit assumption in the gain time calculation that the light propagates inside the sphere during the scattering process. In reality, a substantial fraction of the electromagnetic energy of the resonance $(7.1 \%$ in our case) is stored in the evanescent field just outside the sphere. ${ }^{21}$

In our experiments we used a suspension of polystyrene (PS) microspheres in water (G1000, Duke Scientific Corporation; the measured refractive index of water is 1.3325). The manufacturer specifies a mean radius of $5.05 \mu \mathrm{m} \pm 5 \%$ (standard deviation). The microspheres are doped with $2 \%$ fluorescent dye with a broad emission band $\lambda_{0}=480-540 \mathrm{~nm}$. A single microsphere is trapped with optical tweezers and lifted by several times its diameter from the surface of the glass capillary. The excitation light source is a pulsed Coherent Infinity-XPO emitting at $470 \mathrm{~nm}$ with a repetition rate of $50 \mathrm{~Hz}$, a pulse duration of $\sim 3 \mathrm{~ns}$ and a maximum pulse energy of $15 \mathrm{~mJ}$ per pulse, which is attenuated to $100 \mathrm{~nJ}$ at the sample. The excitation light is slightly defocused on the sphere, so that the whole sphere is illuminated. The light emitted by the dye is collected through a water-immersed objective $(\mathrm{NA}=1.2)$ and detected by an intensified charge coupled device (ICCD) attached to a spectrograph, which has a Gaussian response function with a width of $4 \mathrm{~cm}^{-1}$. One count on the ICCD corresponds roughly to one emitted photon. Below threshold we averaged over 10,000 pulses and above threshold 500 pulses to obtain a good signal-tonoise ratio. The emission spectra are corrected for background due to stray light and normalized to the peak intensity of the $\mathrm{TE}_{95}$ mode. For a determination of the absorption index of the spheres, we have measured the emission linewidths with low-power cw excitation. We found a value for the absorption index of $(1.5 \pm 1.5) \times 10^{-5}$.

In Fig. 1(a) we present the measured emission spectrum for low pumping power $(1 \mathrm{~nJ} /$ pulse on the sample). The MDRs are clearly observed and are characterized by their mode numbers and additionally labeled either TE or $\mathrm{TM}^{7}$ (transverse magnetic). The theoretical curve in Fig. 1(a) that is fitted to the experimental data was convolved with the Gaussian response function of the spectrograph and has been normalized to the peak intensity of the $\mathrm{TE}_{95}$ mode. Fit parameters were $m_{I}^{\prime}$, the real refractive index, and $a$, the radius of the sphere. We found the best fit for $m_{I}^{\prime}=1.583 \pm 0.001$ and $a=5.29 \pm 0.01 \mu \mathrm{m}$. The value for $m_{I}^{\prime}$ corresponds within $1 \%$ to reported measurements on pure PS microspheres. ${ }^{22}$ As can be seen in Fig. 1(a), our measured and calculated emission spectra are in good agreement.

In Fig. 1(b) we show the experimental emission spectrum of the same microsphere at a pulse energy of $100 \mathrm{~nJ} /$ pulse, which is above threshold. Three intense modes are visible in the spectrum with an intensity of up to 30 times the intensity of the fluorescent background and a width smaller than the width of the peaks below threshold. We have verified that all three modes $\left(\mathrm{TE}_{95}, \mathrm{TM}_{95}\right.$, and $\left.\mathrm{TE}_{96}\right)$ are above threshold and show similar behavior and that their widths remain constant upon more pumping. From now on, we discuss only the results for the $\mathrm{TE}_{95}$ mode, which has the lowest threshold. We explore the data only up to this threshold. In this regime no other modes are lasing, and the intensities of the different modes below threshold are low enough that mode competition may be ignored.

We verify the lasing behavior of $\mathrm{TE}_{95}$ by considering the integrated intensity in the laser mode at $19400 \mathrm{~cm}^{-1}$ versus the luminance, shown in Fig. 2.

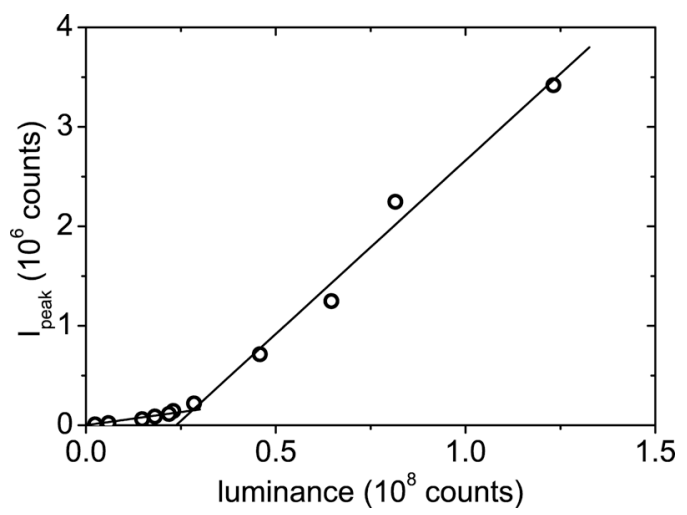

Fig. 2. Measured integrated intensity in the $\mathrm{TE}_{95}$ mode versus luminance of a sphere with gain (the lines are a guide to the eye). The laser threshold is clearly visible. 


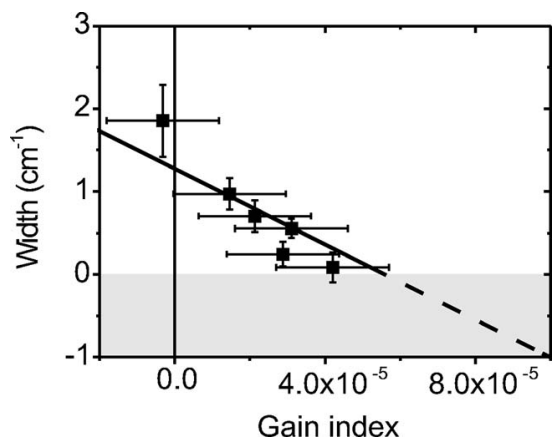

Fig. 3. Width of the $\mathrm{TE}_{95}$ mode versus the gain index. The line represents the calculated width from Mie theory. The squares are data points from fits to the measured spectra. The error bars for the width are taken from the fits; the error bars for the gain index are taken from cw experiments. The gray area indicates the nonphysical area. Above the laser threshold the measured data points are not presented, as the gain saturates in the experiment.

The luminance is defined as the integrated intensity in the complete emission spectrum and is proportional to the pump power absorbed by the microsphere. We see the expected threshold behavior for a laser ${ }^{23}$ and obtain from this graph the laser threshold in terms of luminance.

We now compare the prediction of our model with our experiments. For the measured emission spectra (below and above threshold), we determined the Lorentzian width of the $\mathrm{TE}_{95}$ mode by fitting it with a Voigt profile, with the Gaussian linewidth set equal to the response of the ICCD camera. We know the theoretical width from EMTG as a function of the gain index, and the experimental widths as a function of the luminance. A linear relation is assumed between the luminance and the gain index, an adequate assumption at low values of the gain index. Two points fix their relation: at threshold we know both the gain index (from the fit to our data) and the luminance (see Fig. 2); at zero luminance the gain is zero and the (negative) gain index is given by the absorption of the sphere.

In Fig. 3 we present the theoretical as well as the experimentally observed width of the $\mathrm{TE}_{95}$ mode as a function of the gain index. Below threshold the data are in good agreement with EMTG. Above threshold we measured a very small linewidth as expected from laser theory with gain dynamics. ${ }^{23}$ One could question the assumption of a homogenous gain, as the pump light will also undergo Mie scattering. However, given the large absorption index due to the dye molecules inside the sphere, Mie resonances are washed out at the pump wavelength.

In conclusion, we observed that in the case of spheres with time-independent gain index EMTG gives physical results up to a certain threshold. We identify this threshold as the laser threshold. Above this threshold, an extended, time-dependent Mie theory (e.g., with pump and gain dynamics) is needed to describe the laser behavior correctly. In our experiments we have measured narrowing of the $\mathrm{TE}_{95}$ mode as a function of the gain index up to the lasing threshold, confirming both the validity of EMTG below threshold and our interpretation of the point of the divergence of the scattering coefficients as the laser threshold.

This work is part of the research program of the Stichting voor Fundamenteel Onderzoek der Materie (FOM), which is financially supported by the Nederlandse Organisatie voor Wetenschappelijk Onderzoek (NWO). K. van der Molen's e-mail address is k.l.vandermolen@utwente.nl.

\section{References}

1. M. Kerker, ed., Selected Papers on Light Scattering, Parts 1 and 2 (SPIE, 1988).

2. H.-B. Lin, J. D. Eversole, and A. J. Campillo, J. Opt. Soc. Am. B 9, 43 (1992).

3. M. Kuwata-Gonokami, K. Takeda, H. Yasuda, and K. Ema, Jpn. J. Appl. Phys. 31, L99 (1992).

4. V. Sandoghdar, F. Treussart, J. Hare, V. LefévreSeguin, J.-M. Raimond, and S. Haroche, Phys. Rev. A 54, R1777 (1996).

5. A. J. Campillo, J. D. Eversole, and H.-B. Lin, Phys. Rev. Lett. 67, 437 (1991).

6. M. Cai, O. Painter, K. J. Vahala, and P. C. Sercel, Opt. Lett. 25, 1430 (2000).

7. C. F. Bohren and D. R. Huffman, Absorption and Scattering of Light by Small Particles (Wiley, 1983).

8. H. Chew, J. Chem. Phys. 87, 1355 (1987).

9. H. Chew, Phys. Rev. A 38, 3410 (1988).

10. J. Ripoll, C. M. Soukoulis, and E. N. Economou, J. Opt. Soc. Am. A 21, 141 (2004).

11. V. V. Datsyuk, J. Opt. Soc. Am. B 19, 142 (2002).

12. B. A. Hunter, M. A. Box, and B. Maier, J. Opt. Soc. Am. A 5, 1281 (1988).

13. M. Kerker, Appl. Opt. 18, 1180 (1979).

14. N. G. Alexoupoulos and N. K. Uzunoglu, Appl. Opt. 17, 235 (1978).

15. M. Kerker, Appl. Opt. 17, 3337 (1978).

16. K. J. Vahala, Nature 424, 839 (2003).

17. J. Heinrichs, Phys. Rev. B 56, 8674 (1997).

18. C. W. J. Beenakker, J. C. J. Paaschens, and P. W. Brouwer, Phys. Rev. Lett. 76, 1368 (1996).

19. H. M. Lai, C. C. Lam, P. T. Leung, and K. Young, J. Opt. Soc. Am. B 8, 1962 (1991).

20. H. M. Nussenzveig, J. Math. Phys. 10, 82 (1969).

21. A. Bott and W. Zdunkowski, J. Opt. Soc. Am. A 4, 1361 (1987).

22. X. Ma, J. Q. Lu, R. Scott Brock, K. M. Jacobs, P. Yang, and X.-H. Hu, Phys. Med. Biol. 48, 4165 (2003).

23. A. E. Siegman, Lasers (University Science, 1986). 